\title{
Machine Learning Based Image Feature Recognition and Clinical Nursing of Children's Rheumatoid Arthritis-Related Lung Injury
}

\author{
Linyan Li \\ Department of Pediatric Nephrology and Rheumatology, No. 1 People's Hospital, Chenzhou 42300, Hunan, China \\ Correspondence should be addressed to Linyan Li; 151120500@ecupl.edu.cn
}

Received 13 June 2021; Revised 29 June 2021; Accepted 14 July 2021; Published 22 July 2021

Academic Editor: Gustavo Ramirez

Copyright (C) 2021 Linyan Li. This is an open access article distributed under the Creative Commons Attribution License, which permits unrestricted use, distribution, and reproduction in any medium, provided the original work is properly cited.

\begin{abstract}
This work was aimed at investigating image feature recognition and clinical nursing of children's rheumatoid arthritis- (CRA-) related lung injury under maximum correlation minimum redundancy algorithm of machine learning. In this study, $18 \mathrm{children}$ with CRA in the hospital were selected as the rheumatoid group to explore the nursing method, and 18 healthy children were selected as the control group. The maximum correlation minimum redundancy algorithm of machine learning was compared with the information gain algorithm and the Fisher score algorithm and applied in computed tomography (CT) images of 18 CRA children. The classification accuracy of the algorithm in this study $(94.52 \%)$ was higher than that of the information gain algorithm $(88.64 \%)$ and Fisher score algorithm $(81.24 \%)$. CT alveolitis score $(2.35 \pm 0.72$ points) of children from the rheumatoid group was markedly higher than that of the control group ( $1.21 \pm 0.24$ points) $(t=2.147$ and $P<0.05)$. The nitric oxide level ( $14.00 \mathrm{ppb})$ of children from the rheumatoid group increased greatly compared with the control group $(10.00 \mathrm{ppb})(P<0.05)$. CRA can cause a decline of lung function in children, while the nitric oxide level exhaled by children can assess the activity of RA. In addition, adopting active nursing methods can help children get better.
\end{abstract}

\section{Introduction}

Children's rheumatoid arthritis (CRA) is a systemic connective tissue disease, mainly manifested by fever, joint swelling, and pain, often accompanied by rash and the enlargement of liver and spleen lymph nodes. The lungs will be involved because the lungs contain a large number of capillaries and connective tissues, and then the serosal membrane, lung parenchyma, and lung airways will be also involved, resulting in lung nodules and bronchitis [1]. Wojcik et al. [2] pointed out that the pathogenesis of CRA was that the citrulline in the lungs would be immune under the condition of oxidative stress, which induced successive immune responses in other organs or tissues containing citrulline. Besides, the symptoms appeared, particularly in the joints. Common CRA includes systemic lupus erythematosus (SLE), juvenile dermatomyositis (JDM), and systemic-onset juvenile idiopathic arthritis (SOJIA) [3]. About $0.3 \%$ of the world's population has been plagued by it, and children patients with CRA account for about $0.12 \%$ in
China [4]. In clinical medicine, there are still difficulties in the diagnosis of lung injury. Therefore, it is necessary to identify children with CRA promptly and carry out nursing interventions, to prevent the deterioration of lung injury, which is of positive significance for improving the prognosis of children.

CT is to apply X-rays or $\gamma$-rays to scan a certain part of the human body together with a highly sensitive detector, which is featured with fast scanning time and clear scanned images [5]. In recent years, CT images have been extensively used in the field of medical clinical diagnosis. The injured parts of the human body are scanned by CT, and the disease is determined by image analysis. CT examination has become an indispensable way in the medical field of lung diagnosis [6]. Machine learning is mainly divided into supervised and unsupervised. When performing feature selection, the distinction is mainly based on whether the data set is marked or not [7]. Cruz et al. [8] adopted a machine learning algorithm based on maximum correlation and minimum redundancy to minimize the classification error to 
obtain the optimal feature. Under the condition of unsupervised learning, the minimization of the classification error required the subspace of the feature to show the greatest dependence on segmented images. The maximum correlation minimum redundancy algorithm was applied to minimize the redundancy, thereby classifying CT images and extracting the most effective feature set.

The maximum correlation minimum redundancy algorithm of machine learning was compared with the information gain algorithm and the Fisher score algorithm and applied to the CT images of 18 children with CRA. The objective of this study was to explore the CT imaging features and clinical nursing of CRA-related lung injury.

\section{Materials and Methods}

2.1. General Data. A total of 36 children diagnosed with CRA in our hospital from October 2018 to December 2019 were selected in this study: 18 cases with rheumatoid arthritis as the rheumatoid group and the remaining 18 children as the control group. In the rheumatoid group, there were 7 males and 11 females, and they were $7-12$ years old, with an average age of $10.23 \pm 2.18$ years. In the control group, there were 8 males and 10 females; the age of them was $8-12$ years, with an average age of $10.14 \pm 2.01$ years. The experiment was approved by the medical ethics committee of the hospital, and the patients and their family members knew about the experiment and signed the informed consent forms.

The criteria for inclusion were defined to include children patients who were 7-12 years old; conformed to CRA pathological diagnostic criteria; had diffuse pulmonary lesions in the chest imaging; suffered from abnormal ventilation, slow growth, respiratory failure, and other symptoms found in lung function examination; and had complete case data.

The criteria for exclusion were defined to exclude children patients who had inflammation of some organs or infection of some organs; suffered from organic dysfunction of heart, liver, kidney, and lung; had diseases of the nervous or mental system; suffered from pulmonary bronchial dysplasia; were complicated with tuberculosis, malignant tumor, or other autoimmune diseases; had other genetic diseases; and had incomplete clinical data.

2.2. Research Methods and Diagnostic Criteria. All data of the selected children with CRA were collected, including general data (gender and age), clinical data (medical history and symptoms (joint swelling and joint pain)), and laboratory lung function index (serum C-reactive protein (CRP) and serum complement C3). The 28 -joint disease activity score using erythrocyte sedimentation rate (DAS28-ESR) was employed to score the disease activity of children with CRA.

The diagnostic criteria for CRA were as follows. First, CT examination of the chest showed the imaging manifestations of rheumatoid (ground glass, honeycomb, grid-like degeneration, pleural changes, and thickening of the lobular septum). Second, the lung function examination results revealed restricted lung ventilation disorders. Third, CRA was confirmed by bronchoscopy. Fourth, a surgical lung biopsy was confirmed as CRA. An associate respiratory physician confirmed that one child patient met the above 2 items, and thus the child patient can be diagnosed as CRA.

2.3. DAS28-ESR Clinical Data Collection and Scoring. In this study, an erythrocyte sedimentation rate analyzer (DAS28ESR) was employed to score the activity of joint diseases in 28 children patients with CRA.

(1) Clinical data collection: The age, height, body weight, body mass index (BMI), tender joint count (TJC), swollen joint count (SJC), and visual analogue scale (VAS) of CRA patients were collected and recorded.

(2) The scoring equation of DAS28-ESR is as follows:

$$
\begin{aligned}
\text { DAS28 }- \text { ESR }= & 0.56 \times \text { SQRT }(\text { TJC28 })+0.28 \\
& \times \text { SQRT }(\text { SIJC28 })+0.014 \\
& +0.7 \times \operatorname{In}(\text { ESR }) \times(\text { VAS }) .
\end{aligned}
$$

(3) DAS28-ESR score result classification standard: DAS28-ESR $\leq 2.6$ was classified as the remission period, 2.6 points $<$ DAS28-ESR $\leq 3.2$ points was considered as the low activity period, 3.2 points $<$ DAS28-ESR $\leq 5.1$ points meant the moderate activity period, and DAS2 $8>5.1$ points indicated the high activity period.

\subsection{CT Scanning Examination and CT Examination Scoring} Standards for Children with CRA. In this study, GE Lightspeed VCT 64-slice spiral CT scanner (Philips, USA) was adopted to perform a left and right plain scan of the child's chest. Besides, the patient was scanned from top to bottom. The scan range was from the upper thorax to the diaphragm. The scan parameters were as follows. The layer thickness was $0.625 \mathrm{~mm}$, the scanning interval was $0.5 \mathrm{~mm}$, the pitch was $0.984: 1$, the matrix was $512 \times 512$, the voltage was $120 \mathrm{kV}$, and the current was $220-500 \mathrm{~mA}$.

Alveolitis scoring included the following. 0 points meant that alveolitis did not occur. 1 point indicated that the ground glass shadow area accounted for less than $25 \%$ of the lung lobe. 2 points showed that the ground glass shadow area accounted for $26-50 \%$ of the lung lobe. 3 points revealed that the ground glass shadow area covered $51-75 \%$. 4 points meant that the shadow area of ground glass accounted for more than $75 \%$ of the lung lobes.

Lung interstitial fibrosis scoring was as follows. 0 points represented no occurrence of lung interstitial fibrosis. 1 point revealed that alveolar septum thickened and honeycomb area was below 25\%. 2 points meant that the honeycomb area occupied $26-50 \%$. 3 points showed that honeycomb-shape area was $51-75 \% .4$ points indicated that the honeycomb-shape area was more than $75 \%$.

2.5. Lung Function Detection and Treatment Methods. In this study, a pulmonary function instrument (JAEGER, Germany) was used to evaluate the lung function of children 
patients with CRA in strict accordance with the experimental standards of the American Thoracic Association pulmonary function test by an experienced respiratory associate physician. The observation indexes included forced vital capacity (FVC), forced expiratory volume in one second (FEV1), total lung capacity (TLC), and diffusing capacity of the lung for carbon monoxide (DLCO). Among them, FVC refers to the amount of air exhaled with the maximum strength and the fastest speed after inhaling forcefully; FEV1 is the amount of air that can be exhaled as soon as possible after a maximum inhalation within a certain period; TLC refers to the total amount of gas contained in the lungs after deep breathing, which is mainly used to detect the degree of the airway and the size of lung volume; DLCO refers to unit time and pressure difference; carbon monoxide dispersion in alveolar capillaries is measured to reflect the lung's diffusion function. The 18 children with CRA in this study were treated with different treatment methods. Among them, 3 children with SLE and 4 children with JDM were treated with high-dose methylprednisolone, 2 children with cyclosporine, and 9 children with cyclophosphamide shock therapy. If the child had lung bleeding or renal failure, a ventilator was employed immediately to assist breathing and perform plasma exchange.

2.6. Maximum Correlation and Minimum Redundancy Algorithm Based on Machine Learning. For the dependence between the label and the feature, the feature set $T=\left\{x_{1}, x_{2}, \ldots, x_{n}\right\}$ was set to calculate the maximum dependence between the feature and the target sample, which could be expressed as follows:

$$
\operatorname{Max} B(T, a), \quad B=L\left(\left\{x_{1}, i=1,2, \ldots, n\right\} ; a\right) .
$$

In (2), $n=1$ meant that the characteristic value at the time made $L(T ; a)$ reach the maximum value. When $n>1$, multiple characteristic values could also make $L(T ; a)$ reach the maximum value. In the basic incremental search, features were added one by one, to calculate $n-1$ features $T_{n-1}$. Then, $n$ features were successively introduced into the $L(T ; a)$ calculation. The specific equation was as follows:

$$
L\left(T_{n} ; a\right)=\iint q\left(T_{n} ; a\right) \log \frac{q\left(T_{n} ; a\right)}{q\left(T_{n}\right) q(a)} \mathrm{d} T_{n} \mathrm{~d} a .
$$

In (3), $L\left(T_{n} ; a\right)$ stood for the maximum dependence and $q\left(T_{n} ; a\right)$ expressed the multivariate distribution density function. In actual operation, the maximum dependence was not easy to achieve, the massive data was selected and organized usually based on the most relevant feature selection, and the maximum correlation determination equation was shown in the following.

$$
\operatorname{Max} B(T, a), \quad B=\frac{1}{T} \sum_{x_{i \in T}} L\left(T_{i} ; a\right) .
$$

In (4), $B(T, a), T$, and $L\left(T_{n} ; a\right)$ stood for the correlation, the feature set, and the maximum dependence, respectively. There was a correlation between features; it was easy to show redundant information. What is more, more redundant information appeared if the dependence between features was stronger. Assuming that the correlation between two features was relatively large, the distinguishing ability of the other feature would also be weakened if one feature was deleted. To avoid mutual interference between features, it was necessary to supplement the minimum redundancy based on the maximum correlation. The specific calculation equation is

$$
\operatorname{Min} S(T), \quad S=\frac{1}{|T|^{2}} \sum_{x_{i}, x_{j} \in T} L\left(x_{i}, x_{j}\right) .
$$

The above criteria were combined in order to get the maximum correlation minimum redundancy algorithm of this study. $\psi(B, S)$ was redefined by the above equations, and the maximum correlation and the minimum redundancy were combined and optimized, which could be calculated as follows:

$$
\operatorname{Max} \psi(B, S), \quad \psi=B-S .
$$

In (6), $\psi$ represented the difference between the maximum correlation and the minimum redundancy. The image features were searched according to the above algorithm, and the optimal feature was calculated. In addition, the optimal feature satisfied the following equation:

$$
\operatorname{Max}_{x_{j} \in X-T_{n-1}}\left[L\left(T_{i} ; a\right)-\frac{1}{n-1} \sum_{x_{j} \in X-T_{n-1}} L\left(x_{j}, x_{i}\right)\right] .
$$

When the features were independent of each other, the redundancy can reach the minimum, and the correlation can reach the maximum, to obtain the maximum correlation minimum redundancy algorithm of machine learning in this research finally.

\subsection{Design of Simulation Experiment Platform. MATLAB} R2013A (MathWorks Company, USA) was used to carry out the simulation experiment, a PC with a memory of $1.5 \mathrm{~GB}$ was selected as the experimental platform, and the system was Windows 10 . To verify the classification performance of the maximum correlation minimum redundancy algorithm of machine learning, the feature attributes of children with CRA were applied to this study, and the data used came from the experimental database updated in real-time by UCI, to collect critical features of children with CRA.

Support vector machine (SVM) classifier was suitable for the classification of small samples and had good classification accuracy. The specific steps were as follows.

First, the training set was selected from the database, and the number of samples should not be too large because too much would make the classification results fit, thereby affecting the classification results.

Second, the parameters of the training set were applied by SVM to establish a learning model by learning the training set, and the svmtrain function was employed to learn the training set.

Third, SVM adopted the svmtrain function to test the training set, and the test set samples were detected in the 
form of a matrix. Besides, the test set was divided into test labels and test samples, and the predicted labels were compared with the actual labels to get the classification accuracy rate.

2.8. Statistical Methods. Feature selection was analyzed by SPSS 22.0 statistical software. The data that met the normal distribution were represented by the mean \pm standard deviation $(\bar{x} \pm s)$, and the independent sample $t$-test was used for comparison between groups. The non-normally distributed data were expressed as frequency (\%). Besides, Spearman correlation analysis was adopted to examine the correlation between the two indexes. $P<0.05$ meant that the difference was statistically substantial.

\section{Results}

3.1. Comparison of the Classification of the Three Algorithms. The maximum correlation minimum redundancy algorithm was constructed in this study, compared with the information gain algorithm and the Fisher score algorithm, and applied to the CT images of 18 children with CRA. Figure 1 shows that the classification accuracy of the three algorithms was compared when the features were 6,7 , and 8 . When there were 6 features, the classification accuracy of the information gain algorithm was $87.23 \%$, the classification accuracy of the Fisher score algorithm was $79.87 \%$, and the classification accuracy of the maximum correlation minimum redundancy algorithm was $93.64 \%$. When there were 7 features, the classification accuracy of the information gain algorithm, Fisher score algorithm, and the maximum correlation minimum redundancy algorithm was $88.36 \%$, $80.81 \%$, and $93.74 \%$, respectively. In addition, the classification accuracy was $88.64 \%$ (information gain algorithm), 81.24\% (Fisher score algorithm), and 94.52\% (maximum correlation minimum redundancy algorithm) when there were 8 features. The classification accuracy of the maximum correlation minimum redundancy algorithm was higher markedly than the accuracy of the information gain algorithm and the Fisher score algorithm, and the difference was statistically substantial $(P<0.05)$. This indicated that the algorithm of this research had high efficiency in feature selection and can achieve good classification results.

3.2. Comparison of General Information and Symptoms between the Two Groups. There was no marked difference in age and gender between children with CRA and healthy children $(P>0.05)$. Figure 2 reveals that CRP and C3 in serums of subjects from the two groups were compared, with no statistically obvious difference $(P>0.05)$. At the time of the onset of rheumatoid children, 3 cases (16.67\%) of SLE children had clinical symptoms of asthma and cough. Among them, 2 cases of SLE had cough and asthma as the first symptom, while pink bubbling sputum appeared, lung breath sounds were reduced, and bubbling sound was diffuse in 1 case of SLE 7 days after the onset. 4 children $(22.22 \%)$ with JDM had difficulty breathing, coughing weakness, and phlegm sounds in the lungs. 2 cases $(11.11 \%)$ of SOJIA

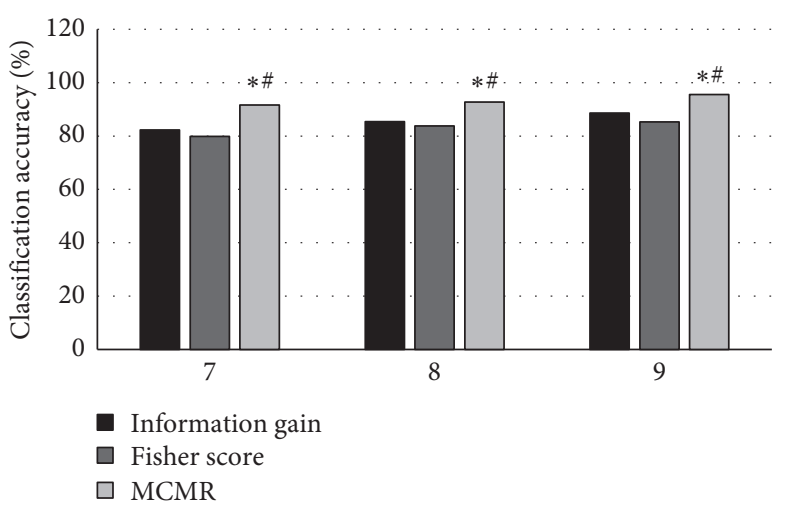

Figure 1: Comparison of the feature selection and classification accuracy of the three algorithms (* the difference was statistically great compared with the information gain algorithm $(P<0.05)$; \#there was a statistically huge difference in contrast to the Fisher score algorithm $(P<0.05)$.

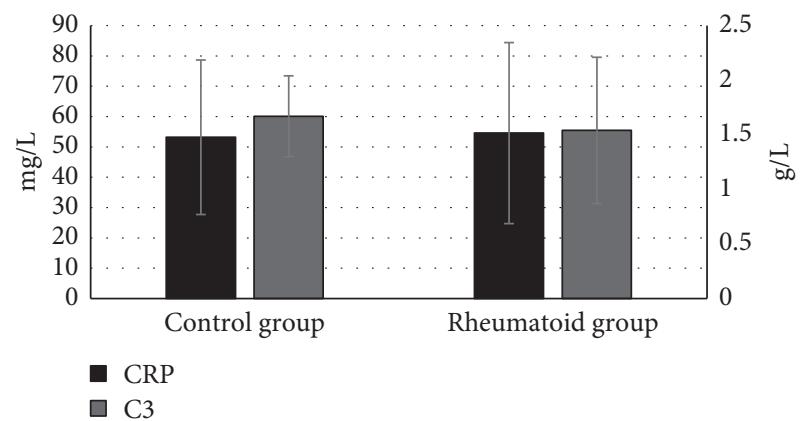

FIgURE 2: Comparison of the serum CRP and C3 of subjects from the two groups.

developed fever and joint pain throughout the body. The other 9 children (50\%) had no obvious respiratory symptoms at the time of the onset (Figure 3).

3.3. CT Imaging Features of Children with CRA. Pleurisy was manifested as pleural effusion of varying degrees. The pleural effusion was crescent-shaped, and the lungs were compressed and moved inward, accompanied by thickening of the pleura. Diffuse interstitial fibrosis was presented as diffuse mesh-like nodular shadows, and ground glass density lesions referred to the lighter density of the lesions in the lung, which were similar to the ground glass. Moreover, the lesions could not cover normal lung vascular images, and normal running and distributed lung blood vessels could be observed in the lesion area (Figure 4). In diffuse interstitial lung lesions, it could be diffusely distributed in both lungs or multisegmented and multilobed in both lungs. The honeycomb-shape image was caused by the distal air cavity expansion, alveolar walls, and lobular septa thickening, which were the late manifestations of lung structural destruction in diffuse interstitial lung fibrosis (Figure 5). Bronchiectasis lost the characteristics of normal bronchiolar branches, the branches were irregular, the bronchial lumen was enlarged, and the tube diameter lost the normal state of 


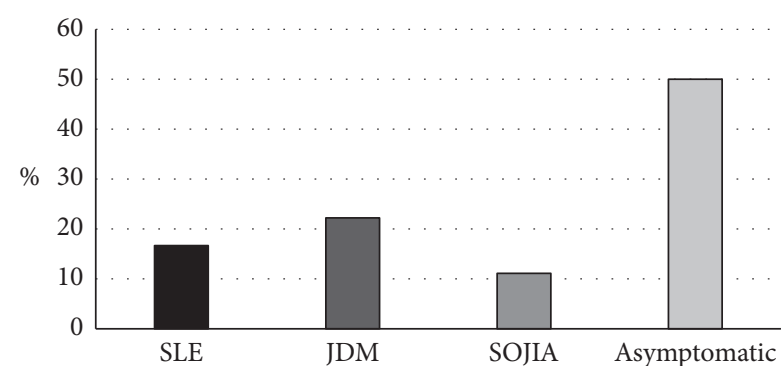

FIGURE 3: Statistics of the proportion of clinical manifestations in children with CRA.

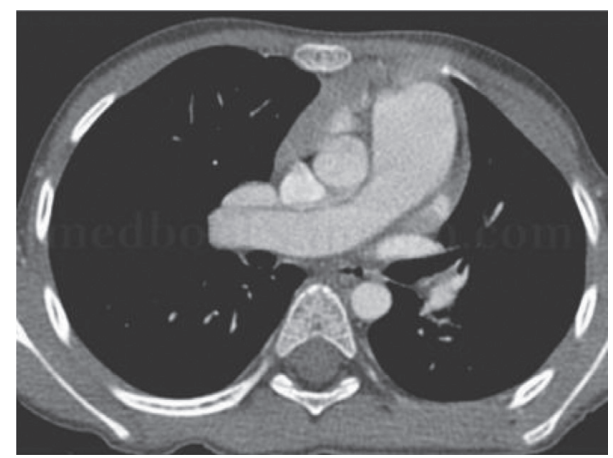

FIgURE 4: An image of multiple ground glass density lesions (the patient was a 1-year-old boy and suffered from SLE).

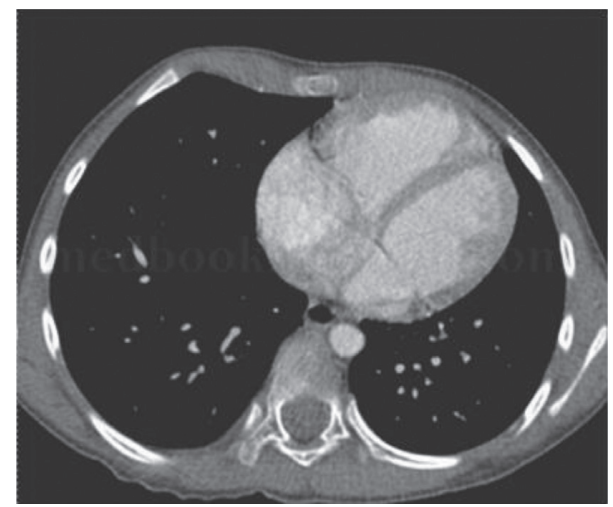

FIGURE 5: An image of diffuse interstitial fibrosis (honeycombshaped image; the patient was a 3-year-old girl with JDM)

changing from thick to thin. Rheumatoid nodules were manifested as nodules located under the pleura, and some could form cavities.

3.4. Statistics of Chest CT Findings in Children with CRA. The 18 enrolled children underwent CT examinations, and all were positive. 8 children $(44.44 \%)$ had the pleural disease: 2 cases with pleural thickening and 6 cases with pleural effusion. There were 12 children $(66.67 \%)$ with interstitial lung disease: 7 children with sheet-like high-density shadows, 2 children with grid-like high-density shadows, 2 children with ground glass, and 1 child with nodular high-density shadow. 7 children (38.89\%) suffered from changes in the lung parenchyma, all of which showed large patches of high density. There were 2 cases $(11.11 \%)$ with localized bullous pneumothorax (Figure 6).

3.5. CT Examination Score and DAS-28ESR Score Results. The scores of CT examinations of subjects from the two groups were compared, suggesting that the CT alveolitis score of children from the rheumatoid group $(2.35 \pm 0.72$ points) was higher than the score of the control group (1.21 \pm 0.24 points), and the difference was statistically obvious $(t=2.147$ and $P<0.05)$. Figure 7 also indicates that the lung fibrosis score of children with CRA (1.68 \pm 1.24 points) was higher than that of the control group ( $0.94 \pm 0.21$ points) $(t=2.071$ and $P<0.05)$. The DAS28-ESR score of children from the rheumatoid group increased extremely compared with the control group $(t=4.251$ and $P<0.05)$ (Figure 8$)$.

3.6. Comparison of Related Indexes of Lung Function of Subjects from the Two Groups. The indexes of lung function of research objects from the two groups were compared, indicating that the FVC of the children from the rheumatoid group was $103.54 \pm 18.65 \%$, which was sharply lower than the FVC of the control group $(126.45 \pm 25.48 \%)(P<0.05)$. The TLC of children with CRA $(105.69 \pm 16.17 \%)$ was steeply lower than that of the control group $(134.36 \pm 36.84 \%)$ $(P<0.05)$. Besides, the DLCO of children from the rheumatoid group $(94.21 \pm 17.43 \%)$ decreased sharply in contrast to the DLCO of the control group $(106.59 \pm 23.41 \%)$, with a statistically obvious difference $(P<0.05)$. Figure 9 also shows that the FEVI of the two groups had no statistically substantial difference $(P>0.05)$. It discloses that the lung function indexes of children with CRA had different degrees of decline compared with healthy children.

3.7. Comparison of the Nitric Oxide Levels of Research Objects from the Two Groups and the Correlation Analysis of Nitric Oxide. The exhaled nitric oxide levels of children from the two groups were compared, and the results are shown in Figure 10. It was found that the nitric oxide level (14.00 ppb) of children from the rheumatoid group was hugely higher than the level of the control group $(10.00 \mathrm{ppb})(P<0.05)$. The level of exhaled nitric oxide in children with CRA was compared with the indexes of FVC, TLC, and DLCO, indicating that there was no statistical significance $(P>0.05)$.

The correlation and related degree of the nitric oxide level with the DAS28-ESR score, alveolar inflammation score, and pulmonary interstitial fibrosis score were analyzed, and the results are shown in Figure 11. The level of nitric oxide was positively correlated with the DAS28-ESR score $(r=0.452$ and $P<0.05)$, nitric oxide level had a positive correlation with alveolitis score $(r=0.245$ and $P<0.05)$, and nitric oxide level was negatively related to lung interstitial fibrosis score $(r=-0.324$ and $P<0.05)$.

3.8. Analysis of the Nursing and Condition Transformation after Treatment of CRA Children Patients. The children's patients were for nursing, to record their breathing rate, diet 


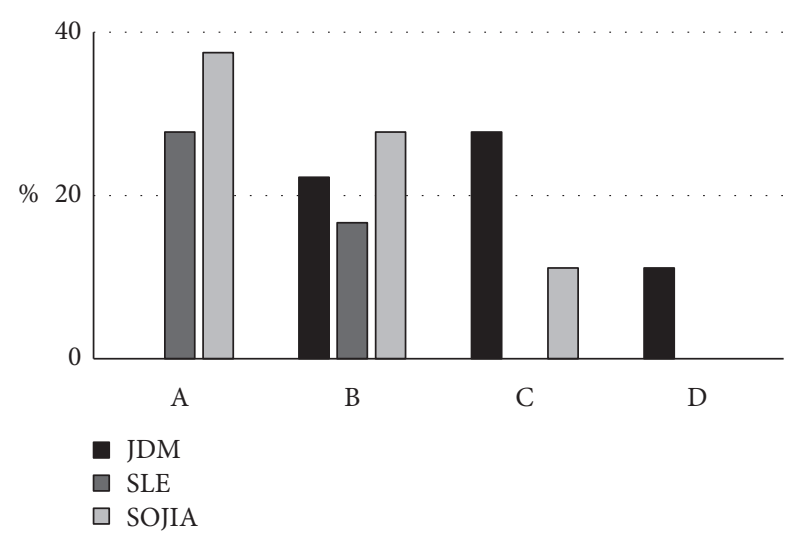

FIgURE 6: Statistics of CT findings of lungs in children with CRA (A: pleural changes; B: lung interstitial changes; C: lung parenchyma changes; D: pneumothorax).

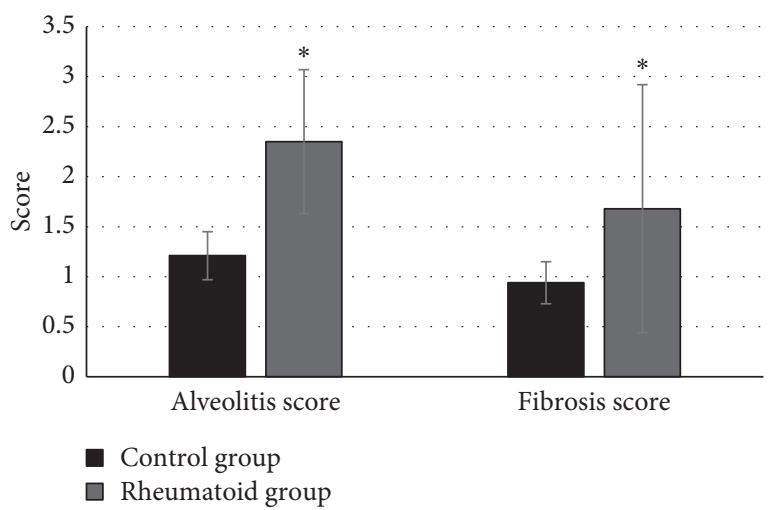

Figure 7: Comparison of the CT examination scoring results of subjects from the two groups $\left({ }^{*} P<0.05\right.$ in contrast to the control group).

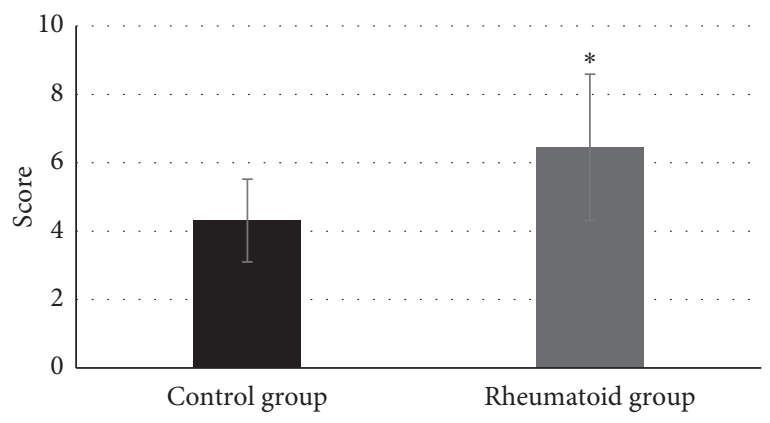

FIGURE 8: Comparison of the DAS28-ESR scores of research objects from the two groups $\left({ }^{*} P<0.05\right.$ in contrast to the control group).

control, doctor-patient communication, psychological evaluation, and condition transformation of children with CRA after nursing and treatment, as shown in Figure 12. It was found that the condition of 15 cases $(83.33 \%)$ was gradually improved under treatment, but 3 cases $(16.67 \%)$ died (Figure 12). Among the deaths, 2 died of acute lung failure, and 1 died of severe lung damage and respiratory failure.

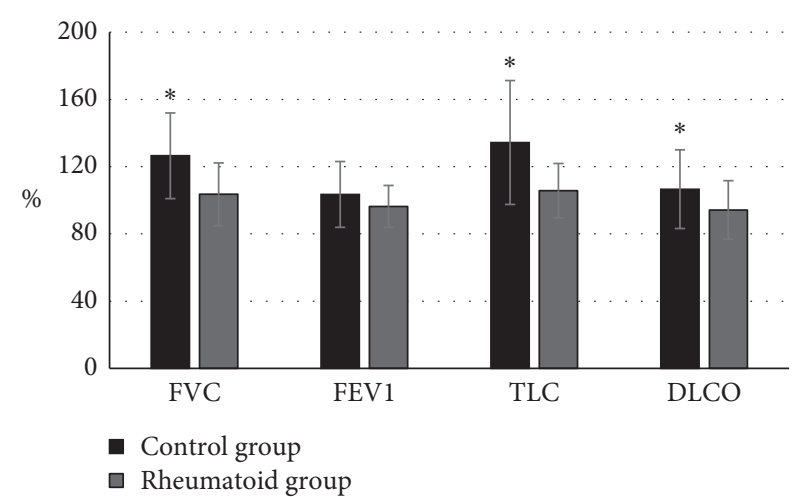

Figure 9: Comparison of the related indexes of lung function of the two groups ( ${ }^{*} P<0.05$ compared with the control group).

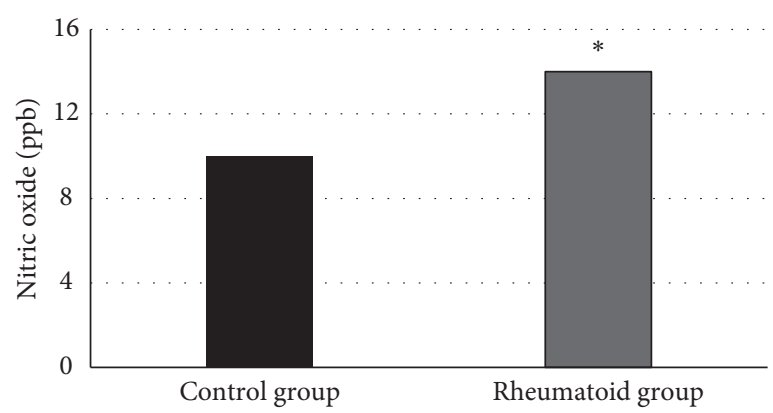

Figure 10: Comparison of the exhaled nitric oxide levels of the two groups $\left({ }^{*} P<0.05\right.$ compared with the control group).

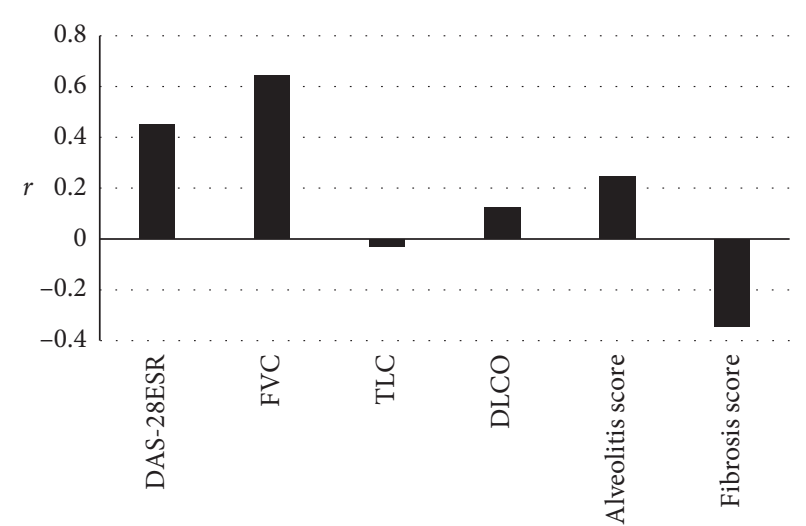

FIGURE 11: Correlation and related degree of the nitric oxide level with the DAS28-ESR score, alveolar inflammation score, and pulmonary interstitial fibrosis score.

\section{Discussion}

The lung lesions of CRA often involve pleura, lung parenchyma, lung interstitium, trachea, and other parts. The clinical manifestations have high invisibility and are not specific during the examination. Therefore, it is easy to have missed diagnosis and misdiagnosis [9]. There are few reports on CRA in China, and most of them are small sample studies. Lee et al. [10] reported that 48 children suffered from 


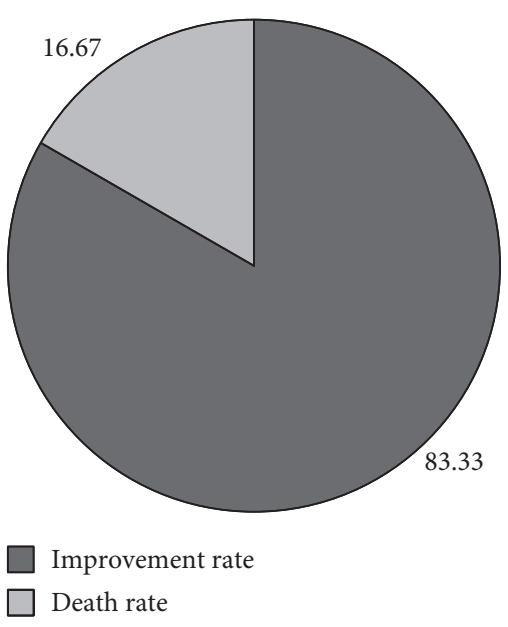

FIgURE 12: Treatment of children with CRA.

JDM, and 3 of them died of respiratory failure due to infection. Ince-Askan et al. [11] thought that children with SLE were prone to serositis, and they were often positive when they were tested for antibodies. Kampschulte et al. [12] pointed out that patients with JDM and systemic lupus erythematosus were prone to lung interstitial fibrosis in CRA. The results of this study revealed that CT examination indicated a wide range of interstitial lesions, accompanied by fibrosis, with a higher mortality rate. One child died due to severe lung injury and respiratory failure, which was consistent with the results of the above research.

It was found that there were differences in the levels of nitric oxide of children from the two groups. The nitric oxide levels of children from the rheumatoid group rose markedly in contrast to the levels of the control group. This was in line with the research findings obtained by Leiva-Salinas et al. [13] by constructing a rat model of lung interstitial fibrosis. The above results were due to the gradual accumulation of bronchial epithelial cells, neutrophils, and inflammatory factors in lung interstitial inflammation, which could promote the production of nitric oxide in the lungs. If the body could repair in time, the level of inflammatory factors would decrease. The degree of lung fibrosis decreases, and the level of nitric oxide in the lungs would be gradually recovered [14]. In this study, the level of nitric oxide in children with CRA was positively associated with the CT alveolitis score, which also suggested that the level of nitric oxide would gradually increase in the stage of alveolitis.

In terms of treatment, high-dose methylprednisolone was used for a shock treatment for critically ill children, and then it was combined with cyclophosphamide for treatment. What is more, critically ill children could be treated with plasma exchange to relieve the conditions. As for nursing, the immunity of children with CRA decreased, so the infection should be actively prevented, the isolation measures should be taken, and the ward should be disinfected regularly. In this study, 2 children suffered from restrictive breathing disorder during treatment, and infection occurred based on lung damage, which gradually improved during treatment. The breathing rate of children should be recorded during nursing, and the position needed to change from time to time to keep breathing smooth. Furthermore, the patients should eat nutritious, light, high-protein, and easily digestible foods. Medical staff should communicate with the children and parents in time to understand the psychological state of the children and make them maintain a positive attitude towards treatment.

\section{Conclusion}

The maximum correlation and minimum redundancy algorithm of machine learning was proposed in this study, compared with the information gain algorithm and the Fisher score algorithm, and applied to the CT images of 18 children with CRA. The algorithm in this study was featured with high efficiency in feature selection and could gain good classification results. CRA could lead to a decline in the lung function of children. The level of nitric oxide exhaled by a child could assess the activity of rheumatoid arthritis disease. Adopting active nursing methods can help the children get better. The limitation of this study is that the sample size is small, which may have an impact on the research results. Later, the sample range will be expanded for further exploration. In short, the results of this study can provide a reliable reference for the clinical diagnosis, treatment, and prognostic nursing of patients with CRA.

\section{Data Availability}

No data were used to support this study.

\section{Conflicts of Interest}

The authors declare no conflicts of interest.

\section{References}

[1] L. R. Jølving, J. Nielsen, U. S. Kesmodel, R. G. Nielsen, N. S. S. Beck, and B. M. Nørgård, "Children born by women with rheumatoid arthritis and increased susceptibility for chronic diseases: a nationwide cohort study," Arthritis Care \& Research, vol. 70, no. 8, pp. 1192-1197, 2018.

[2] S. Wojcik, S. Bernatsky, R. W. Platt et al., "Risk of autism spectrum disorders in children born to mothers with rheumatoid arthritis: a systematic literature review," Arthritis Care and Research, vol. 69, no. 12, pp. 1926-1931, 2017.

[3] G. S. Hazlewood, C. Barnabe, G. Tomlinson, D. Marshall, D. Devoe, and C. Bombardier, "Methotrexate monotherapy and methotrexate combination therapy with traditional and biologic disease modifying antirheumatic drugs for rheumatoid arthritis: abridged Cochrane systematic review and network meta-analysis," BMJ, vol. 353, Article ID i1777, 2016.

[4] P. H. Tsai, K. H. Yu, and I. J. Chou, "Risk of autism spectrum disorder in children born to mothers with systemic lupus erythematosus and rheumatoid arthritis in Taiwan," Joint Bone Spine, vol. 85, no. 5, pp. 599-603, 2018.

[5] H. Ince-Askan, P. R. Mandaviya, and J. F. Felix, "Altered DNA methylation in children born to mothers with rheumatoid arthritis during pregnancy," Annals of the Rheumatic Diseases, vol. 78, no. 9, pp. 1198-1204, 2019.

[6] P. C. Cordeiro, J. P. Guimaraes, and V. A. D. Souza, "Temporomandibular joint involvement in rheumatoid arthritis patients: association between clinical and tomographic data," 
Acta Odontologica Latinoamericana: AOL, vol. 29, no. 3, pp. 123-129, 2016.

[7] M. F. Konig, L. Abusleme, and J. Reinholdt, "Aggregatibacter actinomycetemcomitans-induced hypercitrullination links periodontal infection to autoimmunity in rheumatoid arthritis," Science Translational Medicine, vol. 8, no. 369, 2016.

[8] G. I. Cruz, X. Shao, and H. Quach, "Increased risk of rheumatoid arthritis among mothers with children who carry DRB1 risk-associated alleles," Annals of the Rheumatic Diseases, vol. 76, no. 8, pp. 1405-1410, 2017.

[9] H. Zhu, L. F. Wu, and X. B. Mo, "Rheumatoid arthritis-associated DNA methylation sites in peripheral blood mononuclear cells," Annals of the Rheumatic Diseases, vol. 78, no. 1, pp. 36-42, 2019.

[10] W.-J. Lee, L. Briars, T. A. Lee, G. S. Calip, K. J. Suda, and G. T. Schumock, "Use of tumor necrosis factor-alpha inhibitors in children and young adults with juvenile idiopathic arthritis or rheumatoid arthritis," Pharmacotherapy: The Journal of Human Pharmacology and Drug Therapy, vol. 36, no. 12, pp. 1201-1209, 2016.

[11] H. Ince-Askan, D. A. E. L. T. Van, Y. B. D. Rijke, E. F. C. V. Rossum, J. M. W. Hazes, and R. J. E. M. Dolhain, "Associations between antenatal prednisone exposure and long-term cortisol and cortisone concentrations in children born to women with rheumatoid arthritis: results from a nationwide prospective cohort study," RMD Open, vol. 5, no. 1, 2019.

[12] M. Kampschulte, A. C. Langheinirch, and J. Sender, "Nanocomputed tomography: technique and applications," Röfo: Fortschritte auf dem Gebiete der Rontgenstrahlen und der Nuklearmedizin, vol. 188, no. 2, pp. 146-154, 2016.

[13] C. Leiva-Salinas, B. Jiang, and M. Wintermark, "Computed tomography, computed tomography angiography, and perfusion computed tomography evaluation of acute ischemic stroke," Neuroimaging Clinics of North America, vol. 28, no. 4, pp. 565-572, 2018.

[14] S. Nicolaou and M. F. Mohammed, "Multienergy computed tomography: a new horizon in computed tomographic imaging," Radiologic Clinics of North America, vol. 56, no. 4, pp. 15-16, 2018. 